Como citar: Aguiar, K., Mello, L., \& Andretta, I. (2021). Prejuízos nas habilidades sociais: relações com habilidades de enfrentamento e suporte social. PSI UNISC, 5(2), 142-155. doi: 10.17058/psiunisc.v5i2.15485

\title{
Prejuízos nas habilidades sociais: relações com habilidades de enfrentamento e suporte social
}

\author{
Perjuicio a las habilidades sociales: relación con las habilidades de afrontamiento y el \\ apoyo social
}

\author{
Deficits in social skills: relationships with coping skills and social support
}

Karoline Giele Martins de Aguiar

Universidade do Vale do Rio dos Sinos (UNISINOS), São Leopoldo - RS/Brasil

ORCID: 0000-0001-8310-7273

E-mail: karol.giele@hotmail.com

\begin{abstract}
Luana Thereza Nesi de Mello
Universidade do Vale do Rio dos Sinos (UNISINOS), São Leopoldo - RS/Brasil ORCID: 0000-0003-4139-6681

E-mail: luana.nesi@gmail.com
\end{abstract}

Ilana Andretta

Universidade do Vale do Rio dos Sinos (UNISINOS), São Leopoldo - RS/Brasil ORCID: 0000-0002-5537-5120

E-mail: ilana.andretta@gmail.com

\begin{abstract}
Resumo
Os usuários de crack necessitam de intervenções que contemplem suas especificidades. Trata-se de um estudo correlacional que objetiva avaliar as habilidades sociais (HS), a relação com as habilidades de enfrentamento (HE), e a percepção do suporte social (PSS) de usuários de crack. Participaram 61 usuários de crack em tratamento no CAPS AD III. Os instrumentos utilizados foram: Inventário de Habilidades Social, Inventário de Enfrentamento Antecipatório para Abstinência de Álcool e outras Drogas, e a Escala de Percepção do Suporte Social. Os resultados indicaram associações entre as HS de autocontrole da agressividade com as HE da assertividade e planejamento, autocontrole emocional e afetividade. Além disso as HS em sua totalidade foram correlacionadas com o afeto e com o total das dimensões de PSS. Dessa forma, prejuízos relacionados ao autocontrole em usuários de crack tem associações com as HE e com a PSS.
\end{abstract}

Palavras-chaves: Habilidades sociais; Habilidades de enfrentamento; Cocaína crack; Rede de suporte social.

\section{Resumen}

Los usuarios de crack necesitan intervenciones que aborden sus especificidades. Este es un estudio correlacional que tiene como objetivo evaluar las habilidades sociales (HS), la relación con las habilidades de afrontamiento (HA) y la percepción del apoyo social (PAS) de los usuarios de crack. Participaron 61 usuarios de crack sometidos a tratamiento en CAPS AD III. Los instrumentos utilizados fueron el Inventario de Habilidades Sociales, el Inventario de Afrontamiento Anticipatorio para la Abstinencia del Alcohol y otras drogas, y la Escala de Percepción de Apoyo Social. Los resultados indicaron asociaciones entre las HS del autocontrol de la agresividad: con las HA de asertividad y planificación, autocontrol emocional, y afectividad. Además, las dimensiones totales de HS se correlacionaron con la dimensión de afecto y las dimensiones totales de PSS. quede esta manera, los prejuicios con el autocontrol se asociaron con las HA y la PAS en usuarios de crack.

Palabras clave: Habilidades sociales; Habilidades de afrontamiento; Cocaína crack; Apoyo social.

\footnotetext{
Abstract

Crack users need interventions that contemplate their specificities. This article is a correlational study that aims to evaluate social skills (SS), the relationship with coping skills (CS), and the perception of social support (PSS) in crack users. Paticipated 61 crack users in treatment at CAPS AD III. The instruments used were Social Skills Inventory, Inventory of Anticipatory Training for
} 
Abstinence from Alcohol and Other Drugs, and Social Support Perception Scale. The results indicated associations between the dimension of SS of aggressiveness self-control and $\mathrm{CS}^{\prime}$ dimension of assertiveness and planning, and emotional self-control, and with the dimension of affection. Besides that, the SS in their totality were correlated with affection and the total dimensions of the PSS. Therefore, crack users' deficits in self-control, have associations with CS and with the PSS.

Keywords: Social skills; Coping skills; Crack cocaine; Social support network.

\section{Introdução}

O uso de drogas, no decorrer das últimas décadas, tem crescido de forma considerável e preocupante. Em um estudo realizado pelo Relatório Mundial sobre Drogas (United Nations Office on Drugs and Crime [UNODC], 2020), aproximadamente 269 milhões de pessoas usaram drogas no mundo em 2018, um aumento de $30 \%$ se comparado com o ano de 2009. Além disso, mais de 35 milhões de pessoas sofrem de transtornos associados ao uso de drogas, entre elas o crack. E esse crescimento do consumo de crack também é predominante no Brasil (Bastos, Vasconcellos, De Boni, Reis, \& Coutinho, 2017). Com quase 1,5 milhões de usuários de cocaína e crack em 2019, o Brasil é o maior mercado de cocaína da América do Sul (UNODC, 2020). Segundo a Pesquisa Nacional sobre o uso de crack, realizada em 2012, revelou-se que 366.598 indivíduos fizeram uso de crack e/ou similares, nos últimos seis meses, nas capitais do Brasil, e a região nordeste se destaca por ultrapassar outras regiões, onde cerca de $1,29 \%$ da população usou crack e/ou similares (Bastos \& Bertoni, 2014).

Os usuários de crack tendem a se isolar das situações sociais, a fim de manipular ou esconder o uso da droga, enfraquecendo as relações em termos de afetividade, vinculação e nas interações interpessoais (Horta et al., 2016). Isso ocorre pela inabilidade nas interações sociais e interpessoais, e a literatura aponta que sujeitos com prejuízos nas habilidades sociais, podem estar mais vulneráveis à experimentação do uso de drogas (Schneider, Limberger, \& Andretta, 2016; Silva, 2017).

Desta forma, um repertório satisfatório de Habilidades Sociais (HS), é considerado como fator protetivo ao uso de drogas. Os fatores protetivos são considerados os recursos pessoais e sociais que o indivíduo possui, para lidar com as situações de risco. Estas podem ser expressas de diferentes formas e em diversas situações, são desenvolvidas ao longo da vida (Caballo, 2003). As HS referem-se ao conjunto diversificado de comportamentos sociais do repertório de uma pessoa, que podem contribuir para as suas relações sociais saudáveis e produtivas (Caballo, 2003).

Em um estudo realizado por Schneider e Andretta (2017a), verificaram-se prejuízos nas HS de civilidade nos usuários de crack associados a fatores sociodemográficos. Além disso, as autoras apontam que quanto mais jovem o início de uso da droga e ter familiares envolvidos com o uso de crack são fatores que se relacionaram com os déficits das HS de autocontrole da agressividade em situações aversivas (Schneider \& Andretta, 2017a). Outro estudo realizado com mulheres usuárias de crack aponta que a utilização de drogas no contexto familiar, favorece a compreensão distorcida em relação ao uso de crack e que este está relacionando à resolução de conflitos, podendo favorecer o início do uso (Limberger \& Andretta, 2017).

De forma complementar, em um estudo realizado por Andretta, Limberger e Schneider (2016), ao comparar homens e mulheres usuários de crack, verificou-se déficits nas HS, em ambos os sexos. Nos homens, detectou-se maior necessidade de expressar pensamentos positivos e relação de confiança social, corroborando com estudos já realizados. Já entre as mulheres, observaram-se algumas dificuldades, entre elas, a de como lidar com críticas e baixa autoestima. No decorrer do ciclo vital, ambientes favoráveis contribuem 
para o desenvolvimento saudável e para a construção da autoestima. Quando isso não acontece, pode haver o inverso, a baixa autoestima e, como consequência, diversos sentimentos negativos, sendo um deles a desconfiança em relação à própria capacidade, implicada de forma significativa nas interações sociais, prejudicando o repertório das HS (Bolsoni-Silva \& Marturano, 2002).

Nesse sentido, as habilidades de enfrentamento (HE), podem ser entendidas como o conjunto de estratégias cognitivas e comportamentais relacionadas com a forma que o indivíduo encara os eventos sociais. $\mathrm{O}$ aspecto cognitivo demanda processos mentais, que buscam controlar o comportamento e estratégias comportamentais por envolver uma ação específica, disponível ao indivíduo, que visam lidar com situações adversas relacionadas ao ambiente (Coelho, Sá, \& Oliveira, 2016). O prejuízo das HE e o fracasso nessas interações sociais acabam reforçando o uso da droga, como uma fuga do mal-estar ocasionado pelas relações, tornando-se um ciclo entre o início do tratamento, período de abstinência e a recaída (Rangé \& Marlatt, 2008; Sá, Olaz, \& Del Prette, 2017). Quando o indivíduo possui repertório de $\mathrm{HE}$, relacionadas à afirmação e defesa, situações de risco e de autocontrole da ansiedade, estas favorecem a permanência da abstinência (Coelho et al., 2016; Sá \& Del Prette, 2016). A abstinência pode ser compreendida como o período em que os usuários não retornam aos padrões comportamentais de consumo de droga, e quando isso não acontece ocorre a recaída (Diehl, Cordeiro, \& Laranjeira, 2011).

As HE podem ser compreendidas como o repertório de estratégias cognitivas, que visam possibilitar ao sujeito lidar com situações em que possa se colocar em risco. A ocasião da situação e o contexto que envolve o usuário, dizem respeito aos relacionamentos interpessoais e a comunicação, estas são influenciadas como o sujeito percebe tais relações, interferindo assim, nas HE (Coelho et al., 2016). O fortalecimento das HS de usuários de crack, pode favorecer com que os usuários consigam lidar de forma mais assertiva com a abstinência (Vieira \& Feldens, 2013). Como o usuário percebe a rede de suporte social, propicia a compreensão de como tais HS podem se manifestar em determinados contextos e como favorecerá ou diminuirá a solicitação de suporte social, prevenindo ou provocando à recaída (Caballo, 2003; Carvalho \& Santana, 2018).

Neste sentido, o suporte social pode ser explicado por meio das relações entre o indivíduo e os membros do grupo onde está inserido. Este suporte desenvolve no indivíduo sentimentos positivos de pertença, estima, confiança e zelo. Pesquisas nacionais e internacionais indicam a relevância do suporte social no tratamento de usuários de drogas, visto que o fato do usuário se sentir benquisto $\mathrm{e}$ pertencente a uma rede favorece a adesão ao tratamento, sugerindo fortalecimento positivo da relação com a saúde e promovendo a prevenção de recaída (Carvalho \& Santana, 2018; Borges, del Castillo, Marzo, \& del Castillo-López, 2016).

Diante disso, uma rede de suporte e apoio social pode ser compreendida como um processo de ajuda recíproca entre indivíduos, com o objetivo principal de promover aspectos positivos, abrangendo o lado emocional no âmbito de grupos de pessoas, disponíveis a ofertar o suporte (Amendola, Oliveira, \& Alvarenga, 2011; Cardoso \& Baptista, 2014). Tal conjunto de sistemas e de pessoas que compõem elos de relacionamentos é recebido e percebido pelo indivíduo, envolvendo sentimentos de autonomia, pertença, afetividade e auxílios de ordem material, mas que nem sempre são recíprocos (Cardoso \& Baptista, 2014; Juliano \& Yunes, 2014).

A percepção do suporte social, como fator protetivo e regulador, aborda a qualidade do suporte recebido e percebido na perspectiva do indivíduo. Este suporte relaciona-se não apenas na dimensão do construto do suporte social, mas na qualidade da afetividade, das relações interpessoais, do auxílio material, na ajuda do enfrentamento de problemas e como se dá a interiorização dos sentimentos pelo indivíduo (Cardoso \& Baptista, 2015). Em 
pesquisas realizadas sobre rede de suporte social, observa-se a necessidade de melhor compreensão da percepção dos usuários de crack, vislumbrando a identificação de fatores protetivos e reguladores (Cardona \& Eliana 2014; Cavalcante et al., 2012).

Nesta perspectiva, as HS podem interferir como elemento potencializador ou minimizador das relações interpessoais do usuário e a sua rede de suporte social (Cavalcante et al., 2012; Sá, Olaz \& Del Prette, 2017). Com a problemática do uso de crack no contexto nacional, uma melhor compreensão sobre HS articuladas à percepção do indivíduo acerca de sua rede de suporte social, pode contribuir como estratégias que estimulem relacionamentos interpessoais favoráveis. Além disso, poderá possibilitar o enfrentamento de situações de risco e a manutenção da abstinência, principalmente se levado em consideração os aspectos particulares da região onde o indivíduo reside (Cavalcante et al., 2012; Aguiar, Mello, \& Andretta, 2019). Dessa forma, o objetivo deste estudo é avaliar entre si se há relação entre três variáveis: prejuízos das HS HE antecipatórias para abstinência e a percepção do suporte social de usuários de crack, do Sul do Maranhão.

\section{Método \\ Delineamento}

Trata-se de estudo transversal, quantitativo e correlacional (Sampieri, Collado, \& Lucio, 2013).

\section{Participantes}

A amostra foi constituída inicialmente por 70 participantes em tratamento no CAPS AD III, no Sul do Maranhão. Após verificar os critérios de inclusão, 61 participantes foram incluídos neste estudo. Para isto, levou-se em consideração que o participante apresentasse condições físicas, cognitivas e estivessem em abstinência. Foram excluídos nove participantes por demonstrarem sintomas de dificuldades cognitivas para entendimento dos instrumentos desta pesquisa. Estes foram avaliados pela equipe do serviço e pela pesquisadora.

\section{Instrumentos \\ Questionário de dados sociodemográficos e padrão de uso de drogas}

Este instrumento foi desenvolvido pelo grupo de pesquisa Intervenções Cognitivo Comportamentais: Ensino e Pesquisa (ICCep) e objetiva investigar dados sociodemográficos como: sexo, idade, tempo de abstinência e dados familiares (Limberger \& Andretta, 2017; Schneider \& Andretta, 2017a).

\section{Inventário de Habilidades Sociais (IHS-Del- Prette)}

Desenvolvido por Del Prette e Del Prette (2014), objetiva caracterizar o desempenho social e das interações dos sujeitos em diferentes situações (trabalho, escola, família e cotidiano). É um inventário com escala tipo Likert de cinco pontos ( 1 - nunca; 5 - sempre) composta por 38 itens $(\alpha=.84)$. A análise fatorial revelou uma estrutura de cinco fatores que reúnem habilidades sociais de: F1 Enfrentamento/Autoafirmação com Risco; F2 Autoafirmação de afeto positivo; F3 Conversação e Desenvoltura Social; F4 Autoexposição a Desconhecidos e Situações Novas; e F5 - Autocontrole da Agressividade em Situação Aversiva (Del Prette \& Del Prette, 2014).

\section{Inventário de Habilidades de Enfrentamento Antecipatório para Abstinência de Álcool e Outras Drogas (IDHEA-AD)}

Desenvolvida por Sá e Del Prette (2014), avalia o repertório de habilidades de enfrentamento antecipatório em um conjunto de situações específicas ao contexto do Transtorno por Uso de Substâncias. É composto por 30 itens (situações) os quais o sujeito responde em uma escala do tipo Likert de quatro pontos ( 1 - nunca; 4 - sempre). $O$ IDHEA-AD avalia três fatores, F1 Assertividade e Planejamento para Situações de Alto Risco de Consumo de Substâncias, F2 Expressão Emocional de Sentimentos Positivos 
para a Manutenção da Abstinência e F3 Autocontrole Emocional em Situações adversas. Apresentou confiabilidade interna adequada $(\alpha=.88)$.

\section{Escala de Percepção do Suporte Social - versão adulta (EPSUS-A)}

Desenvolvida por Cardoso e Baptista (2014) avalia o quanto o indivíduo percebe as relações sociais em termos de afetividade, interações, auxílios de ordem prática no processo de tomada de decisão e enfrentamento de problemas. É composta por 36 itens, escala tipo Likert de quatro pontos (1 - sempre; 4 nunca). As dimensões avaliadas são: D1 Afetivo, D2 - Interações Sociais, D3 Instrumental, e a D4 - Enfrentamento de Problemas. O instrumento apresentou alfa de Cronbach de .93). Em relação às interpretações do instrumento, a pontuação da EPSUS-A não possui nota de corte, e os resultados podem variar entre zero e 108, sendo que, quanto maior a pontuação, maior a percepção de suporte social (Cardoso \& Baptista, 2014).

\section{Procedimento de Coleta de Dados}

Os dados foram coletados no CAPS AD III, instituição vinculada ao Sistema Único de Saúde (SUS), em indivíduos que estavam em tratamento e frequentavam a instituição com regularidade, seguindo o Projeto Terapêutico Singular, em uma cidade ao Sul do Maranhão. A coleta foi realizada de forma individual, exclusivamente pela pesquisadora, em um único momento com cada participante, em sala cedida pela instituição, com duração média de duas horas.

\section{Procedimentos Éticos}

Este estudo foi aprovado pelo Comitê de Ética e Pesquisa (CEP) da Universidade do Vale do Rio dos Sinos (UNISINOS), sob parecer de número 2.593.600, estando de acordo com as diretrizes e os preceitos da Resolução $n^{\circ}$ 510/2016 do Conselho Nacional de Saúde. Após a aprovação do CEP, a instituição que já havia emitido a carta da anuência para a aprovação junto ao CEP, foi contatada para dar início à coleta dos dados. Os participantes foram convidados, de forma individual e receberam explicação sobre a pesquisa e as questões éticas. Os que decidiram participar da pesquisa assinaram o Termo de Consentimento Livre e Esclarecido (TCLE), o qual descrevia os procedimentos de sigilo da pesquisa e da guarda do material.

\section{Análise de Dados}

Os dados foram analisados pelo Statistical Package for the Social Sciences (SPSS), versão 25.0, adotando o nível de significância de $5 \%(\mathrm{p} \leq .05)$. Inicialmente foram realizadas as análises descritivas contemplando frequências, porcentagens, média, mediana e desvio padrão da amostra. Posteriormente, verificaram-se a distribuição dos dados pelo teste de Kolmogorov-Smirnov com correção de Lillifors. A partir disso, definiu-se a utilização de testes não paramétricos, visto que os dados das três escalas (IHS, IDHEA-AD e EPSUS-A) não satisfaziam as condições para uso de um teste paramétrico. Para a interpretação das correlações de Spearman, foi utilizada a mensuração sugerida por Dancey e Reidy (2005) em que de 1 a .3 a correlação é fraca, de .4 a .6 é moderada e de .7 a 1 é forte.

\section{Resultados}

Os resultados indicam correlação moderada à fraca entre as dimensões do IHSDel-Prette e as HE do IDHEA-AD para abstinência de drogas. O mesmo se verifica entre as dimensões do IHS-Del-Prette e as dimensões da percepção do suporte social (EPSUS-A). Constata-se que a dimensão do autocontrole da agressividade em situação aversiva (F5) atingiu correlação positiva moderada com a dimensão Total do IDHEA$\mathrm{AD}\left(\mathrm{r}_{\mathrm{s}}=.427 ; p=.01\right)$. Obteve-se também correlação positiva fraca entre: autocontrole da agressividade em situação aversiva (F5) e assertividade e planejamento para situações de alto risco de consumo de substâncias $(\mathrm{F} 1)\left(\mathrm{r}_{\mathrm{s}}=\right.$ $.371 ; p=.01)$; as HS de conversação e desenvoltura social (F3) indicam correlação 
positiva fraca entre a expressão emocional de sentimentos positivos para a manutenção da abstinência (F2) $\left(\mathrm{r}_{\mathrm{s}}=.359 ; p=.01\right)$, já o autocontrole da agressividade em situação aversiva (F5) e autocontrole emocional em situações adversas $(\mathrm{F} 3)\left(\mathrm{r}_{\mathrm{s}}=.334 ; p=.01\right)$.

Encontraram-se correlação positiva e fraca entre as HS de autoafirmação de afeto positivo (F2) com as $\mathrm{HE}$ de expressão emocional de sentimentos positivos para a manutenção da abstinência $(\mathrm{F} 2)\left(\mathrm{r}_{\mathrm{s}}=.290 ; p=\right.$
.05); entre a autoafirmação na expressão do afeto positivo (F2) e a dimensão Total do IDHEA-AD $\left(\mathrm{r}_{\mathrm{s}}=.285 ; p=.05\right)$; autoafirmação na expressão do afeto positivo (F2) e autocontrole emocional em situações adversas (F3) $\left(\mathrm{r}_{\mathrm{s}}=.261 ; p=.05\right)$ e a dimensão Total do IHS-Del-Prette se correlacionou a HE de expressão emocional de sentimentos positivos para manutenção da abstinência $(\mathrm{F} 2)\left(\mathrm{r}_{\mathrm{s}}=.270\right.$; $p=.05)$. As demais associações entre as dimensões do IHS-Del-Prette e IDHEA-AD encontram-se na Tabela 1.

Tabela 1

Relação entre Habilidades Sociais (IHS-Del-Prette) e Habilidades de Enfrentamento Antecipatório para Abstinência de Álcool e Outras Drogas (IDHEA-AD) - posição percentil

\begin{tabular}{|c|c|c|c|c|c|c|}
\hline & \multicolumn{6}{|c|}{ IHS-Del-Prette } \\
\hline & F1 & $\mathrm{F} 2$ & F3 & $\mathrm{F} 4$ & F5 & Total \\
\hline $\begin{array}{l}\text { IDHEA-AD } \\
\text { F1 }\end{array}$ & -.011 & .208 & .024 & .117 & $.371^{* * *}$ & .077 \\
\hline $\mathrm{F} 2$ & .065 & $.290^{*}$ & $.359^{* *}$ & .150 & .181 & $.270^{*}$ \\
\hline F3 & -.102 & $.261^{*}$ & .068 & .027 & $.334^{* *}$ & .118 \\
\hline Total & -.031 & $.285^{*}$ & .146 & .128 & $.427^{* * *}$ & .168 \\
\hline
\end{tabular}

Nota. ${ }^{*} p \leq .05 ;{ }^{* *} p \leq .01$; Valência das correlações: .1 a .3 fraca; .4 a .6 moderada; .7 a 1 forte. Tabela elaborada pelo autor.

No que diz respeito à dimensão Total do IHS-Del-Prette e a dimensão Total do EPSUSA, identifica-se correlação positiva significativa fraca $\left(\mathrm{r}_{\mathrm{s}}=.254 ; p=.05\right) ; \mathrm{a}$ dimensão Total do IHS-Del-Prette e a dimensão do afeto (D1) $\left(\mathrm{r}_{\mathrm{s}}=.227 ; p=.05\right)$. As análises também demonstraram correlações significativas fracas entre as HS, autocontrole da agressividade em situações aversivas (F5) e a dimensão Total do EPSUS-A $\left(\mathrm{r}_{\mathrm{s}}=.365 ; p=\right.$ .01 ); entre o autocontrole da agressividade em situações aversivas (F5) e a dimensão do afeto (D1) $\left(\mathrm{r}_{\mathrm{s}}=.266 ; p=.05\right)$. As demais associações entre as dimensões do IHS-Del-Prette e EPSUS-A encontram-se na Tabela 2.

Tabela 2

Relação entre Habilidades Sociais (IHS-Del-Prette) e Percepção de Suporte Social (EPSUS-A)

\begin{tabular}{|c|c|c|c|c|c|c|}
\hline & \multicolumn{6}{|c|}{ IHS-Del-Prette } \\
\hline & F1 & $\mathrm{F} 2$ & F3 & F4 & F5 & Total \\
\hline \multicolumn{7}{|c|}{ EPSUS } \\
\hline D1 & -.016 & .192 & .148 & .103 & $.266^{*}$ & $.227 *$ \\
\hline D2 & .108 & .059 & -.063 & -.046 & .207 & .106 \\
\hline D3 & -.153 & -.060 & .085 & -.175 & .213 & .021 \\
\hline D4 & .098 & .039 & .088 & .030 & .199 & .188 \\
\hline Total & .062 & .008 & .137 & .048 & $.365^{\text {** }}$ & $.254^{*}$ \\
\hline
\end{tabular}

Nota. ${ }^{*} p \leq .05 ;{ }^{*} p \leq .01$; Valência das correlações: .1 a .3 fraca; .4 a .6 moderada; .7 a 1 forte. Tabela elaborada pelo autor.

Neste sentido, verifica-se a correlação positiva fraca entre: HE de autocontrole emocional em situações adversas (F3) e a dimensão do afeto (D1) $\left(\mathrm{r}_{\mathrm{s}}=.349 ; p=.05\right)$; entre o Fator Total do IDHEA-AD e a dimensão do afeto (D1) $\left(\mathrm{r}_{\mathrm{s}}=.316 ; p=.05\right)$ e a dimensão do enfrentamento de problemas (D4) $\left(\mathrm{r}_{\mathrm{s}}=.300\right.$; $p=.05)$. Constata-se ainda a associação entre a HE de expressão emocional de sentimentos positivos para a manutenção da abstinência (F2) e enfrentamento de problemas (D4) $\left(\mathrm{r}_{\mathrm{s}}=\right.$ $.270 ; p=.05)$. As demais associações entre as 
dimensões do EPSUS-A e IDHEA-AD

encontram-se na Tabela 3.

Tabela 3

Relação entre Habilidades de Enfrentamento Antecipatório para Abstinência de Álcool e Outras Drogas (IDHEA-AD) e a Percepção de Suporte Social (EPSUS-A)

\begin{tabular}{ccccc}
\hline & \multicolumn{3}{c}{ IDHEA-AD } \\
\cline { 2 - 5 } & F1 & F2 & F3 & Total \\
\hline EPSUS-A & & & & \\
D1 & .249 & .081 & $.349^{* *}$ & $.316^{*}$ \\
D2 & -.007 & .004 & .209 & .068 \\
D3 & .100 & .085 & .016 & .113 \\
D4 & .194 & $.270^{*}$ & .151 & $.300^{*}$ \\
Total & .080 & .113 & .238 & .171 \\
\hline
\end{tabular}

Nota. ${ }^{*} \mathrm{p} \leq 0,05 ;{ }^{* *} \mathrm{p} \leq 0,01$; Valência das correlações: .1 a .3 fraca; .4 a .6 moderada; .7 a 1 forte. Tabela elaborada pelo autor.

\section{Discussão}

A partir da avaliação da relação entre as HS, as HE para a abstinência e a rede de suporte social, o estudo identificou prejuízos nas HS de usuários de crack, na maior parte da amostra, da mesma forma que em outros estudos (Horta et al., 2016; Sá \& Del Prette, 2014; Schneider, Limberger, \& Andretta, 2016; Schneider \& Andretta, 2017a, 2017b; Vieira \& Feldens, 2013). As HS têm valor fundamental na relação com as HE, fato esperado por ser construto com base nas HS. Entretanto, propõe-se avaliar as HE em contextos distintos, relacionados ao uso de drogas e às situações de alto risco de consumo. Diante deste cenário e visando avaliar as HE de usuários de drogas, surge o construto de utilizado no IDHEA-AD, como a expectativa de instrumento que se aperfeiçoasse ao contexto do enfrentamento do uso de drogas (Sá \& Del Prette, 2016).

A literatura evidencia que, quanto maior o repertório de HS do indivíduo, mais estratégias cognitivas serão disponibilidades para lidar com potenciais situações que envolvem o risco de drogas (Marlatt \& Donovan, 2009; Rangé \& Marlatt, 2008). Torna-se claro que, prejuízos consideráveis nas HS, como os apresentados, não promovem estratégias cognitivas que favoreçam a abstinência. Para o tratamento do uso de drogas, a fim de se manter a abstinência, faz-se necessário o desenvolvimento das habilidades sociais básicas como, por exemplo, a autoafirmação, a conversação, a autoexposição e o autocontrole, para posteriormente desenvolver a habilidade de enfrentamento (Rangé \& Marlatt, 2008).

Nos primeiros momentos do tratamento, os indivíduos conseguem se manter abstêmios por conta da medicação, mesmo não sendo específica para o crack, amenizando os sintomas clínicos do quadro. Porém, no percurso do tratamento, há situações e/ou contextos de oferta do crack, que solicitam estratégias cognitivas de enfrentamento, as quais o sujeito não dispõe ou há prejuízos consideráveis, fazendo com que haja o retorno ao uso da droga. Diante disto, estudos sobre a terapia cognitivo-comportamental têm possibilitado o desenvolvimento de estratégias focadas no enfrentamento do uso de drogas (Marlatt \& Witkiewitz, 2009; Marquezini, 2019; Melo \& Madalena, 2021; Mendonça \& Coelho, 2018).

Os resultados desta pesquisa indicaram que o autocontrole pode ser entendido como um construto, instável e influenciado por fatores ambientais adversos, sociais, culturais, familiares e de personalidade, sendo a família o primeiro ambiente de desenvolvimento da socialização e dos limites (Sá, 2015). As HS de autocontrole são consideradas como a capacidade do indivíduo em controlar os impulsos, o humor, os comportamentos agressivos, lidar com regras e críticas, sendo desenvolvidas, ao longo da vida do indivíduo e tendo início na infância. 
De acordo com Bandeira, Del Prette, Del Prette e Magalhães (2009), o autocontrole indica associação com a assertividade e a capacidade do indivíduo em planejar estratégias em situações de alto de risco de consumo. A assertividade pode ser compreendida como comportar-se e agir de maneira socialmente adaptativa a determinando contexto ou situação, a fim de estabelecer relações equilibradas (Teixeira, Del Prette, \& Del Prette, 2016). Com isso, estratégias assertivas podem promover a redução de situações estressoras a partir de esforços e da capacidade do sujeito de regular os seus comportamentos. Este fato é corroborado pelo presente estudo, no qual apresenta que quanto maior a habilidade de autocontrole da agressividade, melhor será a habilidade para manter abstinência de drogas ao saber lidar com situações de risco. Dessa forma, a emissão de comportamentos adequados para tal circunstância engloba a capacidade de assertividade ao lidar com situações de risco, pedir desculpas quando oportuno e lidar de forma assertiva com o manejo da raiva (Sá \& Del Prette, 2016).

Os prejuízos nas HS de autocontrole estão em equilíbrio com o controle dos impulsos e a necessidade de satisfação, que resultam na interferência da manutenção da abstinência e nas relações sociais. No que tange às HS de autocontrole e à percepção do suporte social, indica-se que quanto maior percepção dos sentimentos de afeto e carinho recebido, indicados por itens como "me proporcionam momentos agradáveis", "estão ao meu lado quando preciso", "demonstram confiança", "me valorizam", "demonstram carinho por mim", maiores serão as habilidades de autocontrole da agressividade a situações aversivas.

Nesta perspectiva, identificou-se a relação entre $o$ afeto e a cognição, possibilitando a compreensão da simultaneidade uma sobre a outra, o que pode ocasionar distorções cognitivas pelo indivíduo. A compreensão do afeto são fatores pessoais e da personalidade, que podem levar a distorções de crenças que constituem e contribuem para as relações interpessoais do indivíduo e também podem contribuir no processo de autoeficácia e autorregulação (Leme, Del Prette, Koller, \& Del Prette, 2016).

Em estudo realizado por Bueno, Oliveira e Oliveira (2001), sobre HS e traços de personalidade, os dados apontaram que homens têm maiores prejuízos na autoafirmação na expressão de afeto positivo. A amostra da presente pesquisa foi constituída em sua maior parte por homens, indicativo para o prejuízo de autoafirmação na expressão de sentimentos positivos. Este fato justifica-se pela dificuldade destes em lidar com situações que solicitam a expressão de seus aspectos subjetivos, relacionando-se as dificuldades apresentadas em lidar com situações adversas neutras de aproximação, relações básicas do cotidiano, e de receber críticas de forma adequada, assim como elogios pela abstinência.

Os prejuízos na expressão de sentimento positivo se relacionam com as habilidades de autocontrole da raiva. Quando o indivíduo não se consegue expressar seus sentimentos ou aspectos cognitivos, associados ao uso de crack, este terá as mesmas dificuldades ao lidar com a resolução de problemas relacionados a conflitos interpessoais e com habilidade de enfrentamento de risco de uso de substância. Entende-se que o desenvolvimento da autoafirmação, na expressão de sentimentos, ocasiona no indivíduo mais sentimentos de confiança (Coelho et al., 2016).

Observa-se que usuários de crack possuem dificuldades de autocontrole da agressividade associados à baixa percepção do suporte social relacionando ao afeto. Evidenciam-se dificuldades dos usuários de crack em perceberem as relações afetivas positivas (Carvalho \& Santana, 2018; Halpern et al., 2017). Constata-se que, durante o processo do desenvolvimento das HS, a partir das experiências vividas pelo indivíduo, o afeto é internalizado a partir das relações com a família e com o ambiente em que convive (Cabalo, 2003; Del Prette \& Del Prette; 2001). A baixa percepção deste em relação ao suporte 
social indica as dificuldades de relacionamento à rede suporte social (Borges et al., 2016; Cavalcanti, 2018; Souza \& Baptista, 2017). Sendo a rede de suporte social do usuário fundamental para o processo de tratamento, percepções distorcidas da afetividade podem gerar o afastamento da família no cuidar (Pereira, Costa, \& Ronzani, 2016).

Outro estudo realizado por Schneider e Andretta (2017b), sobre HS e usuários de crack, identificou que usuários com início precoce de drogas e/ou que possuem algum membro da família com prejuízos relacionados ao uso de drogas, apresentaram baixos repertórios na habilidade de autocontrole da agressividade a situações aversivas. Este dado indica que o suporte familiar pode ser potencializador no desenvolvimento dos repertórios das HS. Desta forma, promover um ambiente favorável de suporte social pode favorecer a diminuição dos riscos de recaída (Sá, Olaz, \& Del Prette, 2017).

A rede de suporte social é uma ferramenta crucial para a promoção de saúde, de afetividade, de autoestima, de autocontrole e é fortalecedora de enfrentamento de situações estressoras e adesão ao tratamento de drogas (Cavalcante et al, 2012; Gonçalves, Pawlowski, Bandeira, \& Piccinini, 2011). No caso de usuários de crack, torna-se um instrumento a ser incorporado e utilizado como fator essencial para condução terapêutica como forma autorreguladora, ajudando nos momentos de sentimentos de ambivalência e possibilitando a reintegração ao contexto social (Borges et al., 2016).

Neste sentido, as habilidades de conversação e desenvoltura social de usuários de crack, podem favorecê-los ao lidar com situações sociais básicas, alertando o indivíduo diante de situações de alto risco de uso e interferindo nas $\mathrm{HE}$ de expressão de sentimentos positivos para manutenção da abstinência. Um estudo realizado com mulheres usuárias de crack, identificou prejuízo de conversação e desenvoltura social nestas (Limberger \& Andretta, 2017). Este dado enfatiza que indivíduos usuários de crack com bom repertório de conversação e desenvoltura social conseguiram expressar seus sentimentos positivos, como o afeto, na tentativa de se manter abstinente.

A literatura indica ainda que sujeitos com maior percepção de afeto e acolhimento recebido aumentam a probabilidade de receber orientações ou sugestões, indicando abertura ao processo de socialização, potencializando as intervenções terapêuticas (Bandura, Carprara, Barbaraneli, \& Scabini, 2011; Bandura, 2012). A eficácia familiar pode favorecer os processos de desenvolvimento, incluindo assistência instrumental, proporcionando auxílio no enfrentamento de situações de risco (Bandura, 2012), situações essas que podem envolver o uso de drogas ou ocasionando a recaída.

Além disso, quanto maior o repertório de HE do indivíduo ao controlar suas emoções em situações estressoras, maior a possibilidade do desenvolvimento de comportamentos adaptativos às situações de recaída, proporcionando assim a aquisição da autoeficácia (Marlatt \& Donovan, 2009). Torna-se, portanto, mais evidente a percepção do afeto e a percepção do suporte de enfrentamento de situações adversas. Isso revela que indivíduos que possuem maior estratégia para lidar com o risco ao uso de drogas, também possuem recursos cognitivos de sentimentos afetivos e percebem o enfrentamento dos problemas de ordem instrumental, como auxílio para comprar medicação, ou mesmo até a alimentação, por parte da rede de suporte social. Diante dos dados, entende-se que as HS estão correlacionadas às HE e ao uso de drogas, com a percepção do suporte social (Cavalcanti, 2018; Donovam, 2009; Sá \& Del Prette, 2016).

Estudos realizados abordando a percepção do suporte familiar de usuários de crack evidenciaram que eles possuem baixa percepção do suporte ofertado pelos membros da sua família, durante o processo de tratamento para o uso de crack, ocasionando má adesão ao tratamento (Carvalho \& Santana, 2018; Halpern et al., 2017). Este dado evidencia que a rede de suporte social é necessária para $\mathrm{o}$ projeto terapêutico.

\section{.}


literatura indica que sujeitos com maior percepção de acolhimento recebido, aumentam a probabilidade de receber orientações, conselhos ou sugestões que sejam positivas ou críticas, indicando abertura ao processo de socialização nas interações sociais (Bandura et al., 2011; Bandura, 2012). Sendo assim, a afetividade constitui-se como parte essencial do suporte social de usuários de cocaína/crack, tornando-se indispensável no fortalecimento entre os usuários e a sua rede de suporte social, pois esta última alcançará papel regulador e orientador, promovendo a autorregulação do usuário (Conzatti, Rodrigues, Silva, Avila, \& Oliveira, 2016; Fernandes \& Soares, 2018; Gonçalves et al., 2011).

As relações familiares são apontadas pelos usuários como potenciais dificultadores do processo de manutenção da abstinência e, somados a desconfiança da sociedade, reforçam pensamento de uso (Pedrosa, Reis, Gontijo, Teles, \& Medeiros, 2016). Verifica-se que muitos estudos transversais relacionados aos usuários de crack, independente de objetivo, mencionam em suas discussões, a importância do suporte social no tratamento de usuários de crack (Carvalho \& Santana, 2018; Coelho et al., 2016; Fernandes \& Soares, 2018; Schneider \& Andretta, 2017a).

Neste sentido, as associações apresentadas entre as HS de autocontrole da agressividade e as $\mathrm{HE}$ de assertividade e de autocontrole emocional, o total das dimensões das HS e da percepção do suporte social, são evidências do processo e da singularidade em torno do usuário de crack. Os usuários de crack com prejuízos nas HS possivelmente terão dificuldades em manter a abstinência e em fortalecer as relações de suporte social, fatores determinantes para o processo de tratamento.

O período de fissura (craving), somado aos prejuízos das HE, podem facilitar a recaída (Diehl et al., 2011; Horta et al., 2016; Sá \& Del Prette, 2014), fortalecendo as crenças disfuncionais relacionadas aos vínculos afetivos, afetando a rede de suporte social. Nesse sentido, o Treinamento de Habilidades Sociais (THS) vem sendo utilizado para o desenvolvimento de HS (Limberger, TrintinRodrigues, Hartmann, \& Andretta, 2017). A partir dos dados levantados na revisão de literatura e na pesquisa, possibilita-se a construção de discussões sobre métodos e técnicas psicológicas que abordem as temáticas apresentadas, como o THS (Limberger \& Andretta, 2018).

\section{Considerações Finais}

O presente estudo possibilitou destacar as associações entre as HS e as HE como também a percepção do suporte social do usuário de crack. A literatura tem apresentado evidências científicas dos repertórios de HS, associados aos prejuízos relacionados ao uso de drogas, no caso específico o uso de crack. Porém, até o momento, não há estudos que relacionem as habilidades sociais, de enfrentamento antecipatório e a percepção do suporte social, o que justifica estudos envolvendo essas variáveis.

A correlação mútua entre os construtos apresentados proporciona compreensão acerca da complexidade do uso de crack e a dificuldade do usuário em permanecer em abstinência. A partir dos resultados e discussões, parece viável investigar a complexidade do suporte social, incluindo a avaliação das HS da rede de suporte social envolvidas no processo terapêutico, e ainda possibilitar propostas políticas que contemplem, de forma mais incisiva, as redes de suporte. Além disso, deve-se considerar o THS e técnicas de mindfulness como parte integrante do processo terapêutico tanto para o usuário como a rede de suporte social que estará junto ao tratamento. Essa concepção é sustentada por este trabalho e por outros estudos.

As limitações do estudo se referem ao fato da coleta ter ocorrido em apenas uma modalidade de tratamento, não abrangendo os usuários que estão em outras modalidades de terapêutica. No atendimento em CAPS AD há o contato com a rede de suporte social e, em outras formas de tratamento, necessita-se de um período de reclusão. Este distanciamento deve 
ser considerado nas HS e na relação com a rede de suporte social que o usuário tem. Outro fator limitante foi a não avaliação de comorbidade mentais, constituindo-se como indicações para futuros estudos.

Dessa maneira, pretende-se contribuir com a literatura sobre o uso de crack, no contexto nacional, em especial, na região nordeste do Brasil. Além disso, este artigo teve como objetivo incentivar futuros estudos relacionados e servir de provocação a políticas públicas voltadas para melhor assistência aos usuários de drogas, especialmente os usuários de crack. Sugere-se maior tamanho amostral a fim de maior compreensão de suas singularidades e relação com a rede de suporte social, e com isso, desenvolver internções psicoterapêuticasnessa população, como ferramenta de manutenção da abstinência.

\section{Referências}

Aguiar, K. G. M., Mello, L. T. N., \& Andretta, I. (2019). Usuários de crack nordestinos: habilidades sociais, habilidades de enfrentamento e suporte social. Psicologia em Pesquisa, 6(1), 74-82.

Amendola, F., Campos Oliveira, M. A., \& Alvarenga, M. R. M. (2011). Influência do apoio social na qualidade de vida do cuidador familiar de pessoas com dependência. Revista da Escola de Enfermagem da USP, 45(4), 884-889. doi: 10.1590/S0080-62342011000400013

Andretta, I., Limberger, J., \& Schneider, J. A. (2016). Social skills in crack users: differences between men and women. Psicologia: Reflexão e Crítica, 29(1), 45. doi: 10.1186/s41155016-0054-4

Bandeira, M., Del Prette, Z. A. P., Del Prette, A., \& Magalhães, T. (2009) Validação das escalas de habilidades sociais, comportamentos problemáticos e competência acadêmica (SSRS-BR) para o ensino fundamental. Psicologia: Teoria e Pesquisa, 25(2), 271-282. doi: 10.1590/S0102-37722009000200016

Bandura, A. (2012). On the functional properties of perceived self-efficacy revisited. Journal of Management, 38(1), 944. doi: 10.1177/0149206311410606

Bandura, A., Caprara, G. V., Barbaranelli, C., Regalia, C., \& Scabini, E. (2011). Impact of family efficacy beliefs on quality of family functioning and satisfaction with family life. Applied Psychology, 60(3), 421-448. doi: 10.1111/j.1464-0597.2010.00442.x

Bastos, F. I. P. M., \& Bertoni, I. (2014). Pesquisa Nacional sobre o uso de crack: quem são os usuários de crack elou similares do Brasil? quantos são nas capitais brasileiras?. Rio de Janeiro: ICICT.

Bastos, F. I. P. M., Vasconcellos, M. T. L., De Boni, R. B., Reis, N. B., \& Coutinho, C. F. S. (2017). III Levantamento Nacional sobre o uso de drogas pela população brasileira. Ministério da Saúde: FIOCRUZ, Fundação Oswaldo Cruz. Recuperado de https://www.arca.fiocruz.br/handle/icict/34 614

Bolsoni-Silva, A. T., \& Marturano, E. M. (2002). Práticas educativas e problemas de comportamento: uma análise à luz das habilidades sociais. Estudos de Psicologia (Natal), 7(2), 227-235. doi: 10.1590/S1413294X2002000200004

Borges, J. M., del Castillo, J. A. G., Campos, J. C. M., \& del Castillo López, A. G. (2016). Relações entre suporte social, autorregulação e consumo de outras substâncias em adultos portugueses. Actualidades en Psicología, 30(121), 67-75. doi: 10.15517/ap.v30i121.24657

Bueno, J. M. H., Oliveira, S. M. D. S. S., \& Oliveira, J. C. D. S. (2001). Um estudo correlacional entre habilidades sociais e traços de personalidade. Psico USF, 6(1), 31-38. doi: 10.1590/S141382712001000100005

Caballo, V. E. (2003). Manual de avaliação e treinamento das habilidades sociais. São Paulo: Santos.

Cardona, H., \& Eliana, H. (2014). Gerenciamento de caso em usuários de crack: contribuições para o tratamento e 
qualificação da intervenção profissional de um CAPS-AD do DF (Dissertação de Mestrado). Universidade de Brasília, Brasília, Brasil.

Cardoso, H. F., \& Baptista, M. N. (2014). Escala de Percepção do Suporte Social (versão adulta) - EPSUS-A: estudo das qualidades psicométricas. PsicoUSF, 19(3), 499-510. doi: 10.1590/1413$\underline{82712014019003012}$

Cardoso, H. F., \& Baptista, M. N. (2015). Evidência de validade para a Escala de Percepção do Suporte Social (Versão Adulta) - EPSUS-A: um estudo correlacional. Psicologia: Ciência e Profissão, 35(3), 946-958.

Carvalho, M. M. C., \& Santana, S. M. (2018). Uso de crack e suporte familiar: implicações na assistência. Revista Pesquisas e Práticas Psicossociais, 13(1), 1-16.

Cavalcante, L. D. P., Falcão, R. S. T., Lima, H. P., Marinho, A. M., Macedo, J. Q., \& Batista, V. A. B. (2012). Rede de apoio social ao dependente químico: ecomapa como instrumental na assistência em saúde. Revista da Rede de Enfermagem do Nordeste, 13(2). doi:

10.15253/rev\%20rene.v13i2.3920

Cavalcanti, M. G. V. (2018). Habilidades sociais e suporte social em adolescente usuários de maconha e não usuários de drogas (Dissertação de mestrado). Universidade Estadual Paulista Mesquita Filho, Bauru, SP, Brasil.

Coelho, L. R. M., de Sá, L. G. C., \& Oliveira, M. S. (2015). Strategies and Coping Skills of Crack Users in Treatment. Revista de Psicologia da IMED, 7(2), 99-109. doi: 10.18256/2175-5027/psico-imed.v7n2p99109

Conzatti, F., Rodrigues, V. S., da Silva, D. C., de Ávila, A. C., \& da Silva Oliveira, M. (2016). Percepções de usuários de cocaína/crack sobre sua rede de apoio. Aletheia, (49), 48-59.

Dancey, C., \& Reidy, J. (2006). Estatística Sem Matemática para Psicologia: Usando SPSS para Windows. Porto Alegre: Artmed. Del Prette, A., \& Del Prette, Z. A. P. (2014).
Inventário de Habilidades Sociais: manual de aplicação, apuração e interpretação (4a ed.). São Paulo: Casa do Psicólogo.

Diehl, A., Cordeiro, D. C., \& Laranjeira, R. (2011). Dependência Química: prevenção, tratamento e políticas públicas. Porto Alegre: Artmed.

Fernandes, A. M., \& Soares, A. B. (2018). Codependentes de substâncias psicoativas: percepção de suporte social e qualidade de vida. Contextos Clínicos, 11(2), 206-216. doi: $10.4013 /$ ctc.2018.112.06

Gonçalves, R. T., Pawlowski, J., Ruschel Bandeira, D., \& Piccinini, C. A. (2011). Avaliação de apoio social em estudos brasileiros: aspectos conceituais e instrumentos. Ciência \& Saúde Coletiva, 16(3), 1755-1769. doi: 10.1590/S1413-81232011000300012

Halpern, S. C., Scherer, J. N., Roglio, V., Faller, S., Sordi, A., Ornell, F., ... \& Diemen, L. V. (2017). Clinical and social vulnerabilities in crack users according to housing status: a multicenter study in six Brazilian state capitals. Cadernos de Saúde Pública, 33(6), 1-13. doi: 10.1590/0102311x00037517

Horta, R. L., Schäfer, J. L., Coelho, L. R. M., Rodrigues, V. S., Oliveira, M. S. D., \& Teixeira, V. A. (2016). Condições associadas a prejuízo de desempenho em habilidades sociais em uma amostra de conveniência de usuários de crack. Cadernos de Saúde Pública, 32(4). doi: 10.1590/0102-311X00010715

Juliano, M. C. C., \& Yunes, M. A. M. (2014). Reflections on the social support network as a mechanism for the protection and promotion of resilience. Ambiente \& Sociedade, 17(3), 135-154. doi: 10.1590/S1414-753X2014000300009

Leme, V. B. R., Del Prette, Z. A. P., Koller, S. H., \& Del Prette, A. (2016). Habilidades sociais e o modelo bioecológico do desenvolvimento humano: análise e perspectivas. Psicologia \& Sociedade, 28(1). doi: 10.1590/180703102015aop001

Limberger, J., \& Andretta, I. (2017). Desenvolvimento das habilidades sociais na 
vida de mulheres usuárias de crack: estudo de casos múltiplos. Trends in Psychology, 25(4), 1709-1724.

Limberger, J., Trintin-Rodrigues, V., Hartmann, B., \& Andretta, I. (2017). Treinamento em habilidades sociais para usuários de drogas: revisão sistemática da literatura. Contextos Clínicos, 10(1), 99109. doi: $10.4013 /$ ctc. 2017.101 .08

Marlatt, G. A., \& Donovan, D. M. (2009). Prevenção da recaída: Estratégias de manutenção no tratamento de comportamentos aditivos (2a ed.). Porto Alegre: Artmed.

Marlatt, G. A., \& Witkiewitz, K. (2009). Problemas com álcool e drogas. In Marlatt, G. A. \& Donovan, D. M. Prevenção da recaída: Estratégias de manutenção no tratamento de comportamentos aditivos $(2 \mathrm{a}$ ed.). Porto Alegre: Artmed.

Marquezini, F. B. (2019). A terapia cognitivacomportamental aplicada ao tratamento da dependência química. Revista Científica Universitas, 6(3). Recuperado de http://revista.fepi.br/revista/index.php/re vista/article/view/730

Mendonça, G. T., \& Coelho, T. C. (2018). A intervenção em terapia cognitivocomportamental aplicada em dependentes químicos. Caderno Científico Fagoc de Graduação e Pós-Graduação,

3. Recuperado de https://revista.unifagoc.edu.br/index.ph p/caderno/article/view/519

Melo, M. P., \& Madalena, T. S. (2021).

Terapia Cognitivo-Comportamental: uma possibilidade e tratamento para usuários de crack. Cadernos de Psicologia, 2(4).

Recuperado de

https://seer.cesjf.br/index.php/cadernospsic ologia/article/view/2862/1938

Pedrosa, S. M., Reis, M. L., Gontijo, D. T, Teles, S. A., \& Medeiros, M. (2016). A trajetória da dependência do crack: percepções de pessoas em tratamento. Revista Brasileira de Enfermagem, 69(5).

Pereira, L. B., Costa, P. A. H., \& Ronzani, T. M. (2016). As Redes Sociais No Cuidado Aos Usuários de Drogas: Revisão
Sistemática. Psicologia em Estudo, 21(1), 29-39. doi: 10.4025/psicolestud.v21i1.28489

Rangé, B. P., \& Marlatt, G. A. (2008). Terapia cognitivo comportamental de transtornos de abuso de álcool e drogas. Revista Brasileira de Psiquiatria, 30(Supl II), S88-95. doi: 10.1590/S1516-44462008000600006

Sá, E. C. N., (2015). Teoria Geral do Crime: Análise do autocontrole em amostras da população geral e reclusos do sistema prisional (Dissertação de Mestrado). Universidade Federal do Ceará, Fortaleza, CE, Brasil.

Sá, L. G. C., \& Del Prette, Z. A. P. (2014). Habilidades sociais como preditoras do envolvimento com álcool e outras drogas: um estudo exploratório. Interação em Psicologia, 18(2). doi: 10.5380/psi.v18i2.30660

Sá, L. G. C., \& Del Prette, Z. A. P. (2016). Habilidades de enfrentamento antecipatório para abstinência de substâncias: Construção de um novo instrumento de medida. Avances en psicología latinoamericana, 34(2), 351-363.

Sá, L. G. C., Olaz, F. O., \& Del Prette, Z. A. P. (2017). Initial psychometric properties of the Inventory of Anticipatory Coping Skills for Abstinence from Alcohol and Other Drugs. Avaliação Psicológica, 16(2), 176186. doi: 10.15689/AP.2017.1602.08

Sampieri, R. H., Collado, C. F., \& Lucio, P. B. (2013). Metodologia de Pesquisa (3a ed). Porto Alegre: Artmed.

Schneider, J. A., \& Andretta, I. (2017a). Habilidades Sociais de Usuários de Crack em Tratamento nas Comunidades Terapêuticas: Relação com Características Sociodemográficas e de Padrão de Consumo. Revista Colombiana de Psicología, 26(1), 83-98. doi: 10.15446/rcp.v26n1.54032

Schneider, J. A., \& Andretta, I. (2017b). Prejuízos nas habilidades sociais em usuários de crack: Diferenças entre usuários e não usuários. Acta Comportamentalia:

Revista Latina de Análisis del Comportamiento, 25(4), 463-476. 
Schneider, J. A., Limberger, J., \& Andretta, I. (2016). Habilidades sociais e drogas: Revisão sistemática da produção científica nacional e internacional. Avances en psicología latinoamericana, 34(2), 339350. doi: $10.12804 / \mathrm{apl} 34.2 .2016 .08$

Silva, C. M. D. (2017). O uso de drogas na adolescência: identificação de estratégias e proposta de prevenção. Dissertação de especialização, Universidade Federal de Alfenas, Campos Gerais, MG, Brasil.

Souza, M. S., \& Baptista, M. N. (2017).

Associações entre suporte familiar e saúde mental. Psicologia Argumento, 26(54), 207-215. doi:

10.7213/psicolargum.v26i54.19753

Teixeira, C. M., Del Prette, A., \& Del Prette,
Z. A. P. (2016). Assertividade: uma análise da produção acadêmica nacional. Revista Brasileira de Terapia Comportamental e Cognitiva, 18(2), 56-72.

United Nations Office on Drugs and Crime [UNODC]. (2020). World Drugs Report 2020. Vienna: United Nations publication. Recuperado de https://wdr.unodc.org/wdr2020/

Vieira, A. C., \& Feldens, A. C. (2013).

Habilidades sociais, dependência química e abuso de drogas: uma revisão das publicações científicas nos últimos 6 anos. Curso de Especialização em Dependência Química e Promoção da Saúde das Faculdades Integradas de Taquara, Taquara, Brasil.

\section{Dados sobre as autoras:}

- Karoline Giele Martins de Aguiar: Possui graduação em Psicologia (2008) pela Universidade do Oeste Paulista. É Especialista em Saúde Mental e Atenção Psicossocial (2013) pela Faculdade Estácio de Sá, Especialista em Avaliação Psicológica (2019) pelo Conselho Regional de Psicologia (CRP-MA) e Mestre em Psicologia Clínica (2019) pela Universidade do Vale do Rio dos Sinos (UNISINOS). Formação em Avaliação Psicológica. Atualmente é psicóloga do Centro de Atenção Psicossocial Álcool e Drogas (CAPS-AD).

- Luana Thereza Nesi de Mello: Possui graduação em Psicologia (2018) pela Universidade do Vale do Rio dos Sinos (UNISINOS). É Doutoranda em Psicologia pela Universidade do Algarve (UAlg) na Faculdade de Ciências Humanas e Sociais (FCHS) em Portugal desde 2018. É fundadora da Respire - Desenvolvimento Humano.

- Ilana Andretta: Possui graduação em Psicologia (2002) pela PUCRS. É especialista em Psicoterapia Cognitivo-comportamental (2011), Mestre em Psicologia Clínica (2005) pela PUCRS, Doutora em Psicologia (2009) pela PUCRS e terapeuta certificada pela Federação Brasileira de Terapias Cognitivas (FBTC). Possui treinamento avançado em Terapia Cognitiva pelo Beck Institute e em Entrevista Motivacional com William Miller e Theresa Moyers. Atualmente é professora do Programa de Pós-graduação em Psicologia e do curso de graduação em Psicologia da UNISINOS, coordenadora do grupo de pesquisa Intervenções CognitivoComportamentais: estudo e pesquisa (ICCep), bolsista produtividade CNPQ nível 2. É vicecoordenadora eleita do Grupo de Trabalho "Processos, saúde e investigação em uma perspectiva cognitivo-comportamental" da ANPEPP e editora associada da Revista Contextos Clínicos. É psicologa clínica, em consultório privado.

Declaração de Direito Autoral

A submissão de originais para este periódico implica na transferência, pelos autores, dos direitos de publicação impressa e digital. Os direitos autorais para os artigos publicados são do autor, com direitos do periódico sobre a primeira publicação. Os autores somente poderão utilizar os mesmos resultados em outras publicações indicando claramente este periódico como o meio da publicação original. Em virtude de sermos um periódico de acesso aberto, permite-se o uso gratuito dos artigos em aplicações educacionais e científicas desde que citada a fonte conforme a licença CC-BY da Creative Commons. 